\title{
COMPRESSIONS OF GROUP ACTIONS
}

\author{
Z. REICHSTEIN
}

Let $G$ be a finite group. A $G$-variety $X$ is an algebraic variety with a regular $G$-action; $X$ is faithful if every $1 \neq g \in G$ acts non-trivially. I will refer to a dominant $G$-equivariant rational (respectively, regular) map of faithful $G$-varieties as a rational (respectively, regular) compression. All varieties, actions, vector spaces, maps, etc., are assumed to be defined over a fixed algebraically closed base field $k$ of characteristic zero; all varieties are assumed to be irreducible.

I would like to thank V. L. Popov for stimulating discussions and for helpful comments on an earlier draft of this note.

\section{Essential Dimension}

Let $V$ be a faithful linear representation of $G$ and let $d$ be the minimal value of $\operatorname{dim}(X)$, where the minimum is taken over all rational compressions $f: V \rightarrow X$. Note that

(a) (see [1, Theorem 3.1] or [6, Theorem 3.4(b)]) $d$ depends only on the group $G$ and not on the choice of $V$, and

(b) (cf. [6, Proposition 7.1]) in the definition of $d$ we may assume that $X$ is a $G$-invariant subvariety of $V$, i.e., $X$ is the closure of the image of a rational covariant $f: V \rightarrow V$.

The number $d$ is called the essential dimension of $G$ and is usually denoted by $\operatorname{ed}(G)$. This number has interesting connections with the algebraic form of Hilbert's 13th problem, cohomological invariants, generic polynomials and other topics; these connections are described in [1] and [2]. The case where $G=\mathrm{S}_{n}$ is of particular interest. (The notion of essential dimension is also of interest in the context of algebraic groups; see [6] and [7].)

Problem 1. Find ed $(G)$ and, in particular, ed $\left(\mathrm{S}_{n}\right)$.

The value of $\operatorname{ed}(G)$ is known if $G$ is an abelian group; see [1, Theorem 6.1]. For symmetric groups, $\operatorname{ed}\left(\mathrm{S}_{n}\right) \geq[n / 2]$; this is proved, in different ways, in [1, Theorem 6.5(c)], [2, Section 8], [6, Example 12.8] and [7, Section 9]. (For $n=5$ this inequality goes back to Felix Klein [3].) The best known upper bound on ed $\left(\mathrm{S}_{n}\right)$ is ed $\left(\mathrm{S}_{n}\right) \leq n-3$; see $[1$, Theorem $6.5(\mathrm{c})]$, and exact values of ed $\left(\mathrm{S}_{n}\right)$ are only known for $n \leq 6$; see [1, Theorems 6.2 and $\left.6.5(\mathrm{~d})\right]$.

We now consider an analogous notion in the context of regular, rather than rational compressions. Let $V$ be a faithful linear representation of $G$

Z. Reichstein was supported in part by an NSERC research grant. 
and let $D$ be the minimal value of $\operatorname{dim}(X)$, where the minimum is taken over all regular compressions $F: V \longrightarrow X$ from $V$ to a faithful affine $G$-variety $X$. Properties (a) and (b) remain valid in this setting:

(A) $D$ depends only on the group $G$ and not on the choice of $V$ and

(B) in the definition of $D$ we may assume that $X$ is a $G$-invariant subvariety of $V$, i.e., $X$ is the closure of the image of a (regular) covariant $F: V \longrightarrow V$.

Part (B) is an immediate consequence of [5, Theorem 1.7.12]. To prove (A), assume that $V_{1}$ and $V_{2}$ are faithful representations of $G$, and $F_{i}: V_{i} \longrightarrow$ $X_{i}$ is a regular compression with minimal possible values of $\operatorname{dim}\left(X_{i}\right)=D_{i}$, $i=1,2$. We want to show that $D_{1}=D_{2}$. Choose a point $v_{2} \in V_{2}$ such that $F_{2}\left(v_{2}\right)$ has a trivial stabilizer in $X_{2}$. By [5, Theorem 1.7.12] there exists a covariant $\phi: V_{1} \longrightarrow V_{2}$ such that $v_{2}$ lies in the image of $\phi$. Let $X_{1}^{\prime}$ be the closure of the image of $F_{2} \circ \phi: V_{1} \longrightarrow X_{2}$. Then $X_{1}^{\prime}$ is a faithful $G$-variety (because it contains $F\left(v_{2}\right)$ ) and thus, by the definition of $D_{1}$,

$$
D_{1} \leq \operatorname{dim}\left(X_{1}^{\prime}\right) \leq \operatorname{dim}\left(X_{2}\right)=D_{2} .
$$

By symmetry, we also have $D_{2} \leq D_{1}$, so that $D_{1}=D_{2}$, as claimed.

In view of $(\mathrm{A})$, it is natural to write $\mathrm{eD}(G)$ in place of $D$.

Problem 2. Find $\mathrm{eD}(G)$ and, in particular, $\mathrm{eD}\left(\mathrm{S}_{n}\right)$.

Problems 1 and 2 are closely related, because

$$
\operatorname{ed}(G) \leq \operatorname{eD}(G) \leq \operatorname{ed}(G)+1 .
$$

The inequality $\operatorname{ed}(G) \leq \operatorname{eD}(G)$ is obvious from the definition. To prove that $\mathrm{eD}(G) \leq \operatorname{ed}(G)+1$, note that if $f: V \rightarrow V$ is a rational covariant then $F=\alpha f: V \longrightarrow V$ is a regular covariant for some $0 \neq \alpha \in k[V]^{G}$. (That is, $F(v)=\alpha(v) f(v)$ for every $v \in V$.) Then

$$
\operatorname{dim} f(V) \leq \operatorname{dim} F(V) \leq \operatorname{dim} f(V)+1,
$$

and the proof is complete.

\section{INCOMPRESSIBLE VARIETIES}

I will call a $G$-variety $X$ incompressible if every rational compression $X \rightarrow Y$ is a birational isomorphism. (Note that this definition bears some resemblance to the definition of a relatively minimal model in algebraic geometry; cf., e.g., [4, p. 418].)

Problem 3. Classify incompressible G-varieties.

To motivate this problem, I will discuss three simple examples.

Example 4. If $G=\{1\}$ then the only incompressible $G$-variety is a point.

Example 5. If $G=\langle c\rangle$ is a cyclic group of order $n \geq 2$ then there are no incompressible $G$-varieties.

Indeed, assume, to the contrary, that $X$ is an incompressible $G$-variety. Diagonalizing the $G$-action on $k(X)$ (viewed as a finite-dimensional $k(X)^{G}$ vector space), we can find a non-zero rational function $f \in k(X)$ such that 
$f(c \cdot x)=\zeta f(x)$, where $\zeta$ is a primitive $n$th root of unity. Then $x \mapsto f(x)$ is a $G$-compression $X \rightarrow \mathbb{A}^{1}$, where $c$ acts on $\mathbb{A}^{1}$ by $x \longrightarrow \zeta x$. Thus $X$ is birationally isomorphic to $\mathbb{A}^{1}$ (as a $G$-variety). On the other hand, $\mathbb{A}^{1}$ is not incompressible; indeed, $x \longrightarrow x^{n+1}$ is a nontrivial compression $\mathbb{A}^{1} \longrightarrow \mathbb{A}^{1}$, a contradiction.

Example 6. Let $G$ be a finite group that does not embed in $\operatorname{Aut}(X)$ for any curve $X$ of genus $\leq 1$. There are many such groups; see below. Let $X$ be a faithful $G$-curve of the smallest possible genus $g(X)$. (It is easy to see that faithful $G$-curves exist for any finite group $G$; cf., e.g., [1, Remark 4.5].) I claim that $X$ is incompressible. Indeed, suppose that $X \rightarrow Y$ is a $G$-compression. By our assumption, $g(Y) \geq 2$. Then by the Hurwitz formula (see, e.g., [4, Corollary 4.2.4] or [9, Theorem II.5.9]), $g(Y) \leq g(X)$, a contradiction.

To construct a group $G$ that does not embed in $\operatorname{Aut}(X)$ for any curve $X$ of genus $\leq 1$, recall the following classical results on automorphisms of rational and elliptic curves.

- The only finite subgroups of $\operatorname{Aut}\left(\mathbb{P}^{1}\right)=\mathrm{PGL}_{2}$, that are not cyclic or dihedral, are $\mathrm{A}_{4}, \mathrm{~S}_{4}$ and $\mathrm{A}_{5}$; see [3, Chapter 1] or [8, Theorem 2.6.1].

- Let $E$ be an elliptic curve. Then there exists an exact sequence

$$
\{1\} \longrightarrow E \longrightarrow \operatorname{Aut}(E) \longrightarrow H \longrightarrow\{1\}
$$

where $E$ acts on itself by translations (and thus is embedded in $\operatorname{Aut}(E))$ and $|H|=2,4$ or 6 ; see, e.g., [9, Theorems III.10.1 and Proposition X.5.1]. (Note that [9] uses the symbols $\operatorname{Isom}(E)$ and $\operatorname{Aut}(E)$ in place of my $\operatorname{Aut}(E)$ and $H$, respectively.)

It is now easy to see that many finite groups cannot be embedded in $\operatorname{Aut}\left(\mathbb{P}^{1}\right)$ or $\operatorname{Aut}(E)$ for any elliptic curve $E$. Examples of such groups include (i) the symmetric group $\mathrm{S}_{5}$, (ii) the alternating group $G=\mathrm{A}_{6}$, and perhaps more surprisingly (in view of Example 5), the abelian groups (iii) $G=(\mathbb{Z} / p)^{3}$ for $p \geq 5$, (iv) $G=(\mathbb{Z} / 3)^{4}$, and (v) $G=(\mathbb{Z} / 2)^{5}$. Of course, we can also take $G$ to be any group containing a subgroup isomorphic to (i), (ii), (iii), (iv) or (v).

In conclusion, note that Problem 3 can be posed for any algebraic (possibly infinite) group $G$; here a compression should be defined as a dominant map of generically free, rather than faithful, $G$-varieties (cf. [6, Definition $2.15])$.

\section{REFERENCES}

1. J. Buhler and Z. Reichstein, On the essential dimension of a finite group, Compositio Math. 106 (1997), no. 2, 159-179.

2. J. Buhler and Z. Reichstein, On Tschirnhaus transformations, Topics in number theory (University Park, PA, 1997), Kluwer Acad. Publ., Dordrecht, 1999, pp. 127-142. 
3. F. Klein, Lectures on the icosahedron and the solution of equations of the fifth degree, revised ed., Dover Publications Inc., New York, N.Y., 1956, Translated into English by G. G. Morrice.

4. R. Hartshorne, Algebraic geometry, Springer-Verlag, New York, 1977, Graduate Texts in Mathematics, No. 52.

5. V. L. Popov, Sections in invariant theory, The Sophus Lie Memorial Conference (Oslo, 1992), Scand. Univ. Press, Oslo, 1994, pp. 315-361.

6. Z. Reichstein, On the notion of essential dimension for algebraic groups, Transform. Groups 5 (2000), no. 3, 265-304.

7. Z. Reichstein and B. Youssin, Essential dimensions of algebraic groups and a resolution theorem for G-varieties, Canad. J. Math. 52 (2000), no. 5, 1018-1056, With an appendix by J. Kollár and E. Szabó.

8. J. Shurman, Geometry of the quintic, John Wiley \& Sons Inc., New York, 1997.

9. J. H. Silverman, The arithmetic of elliptic curves, Springer-Verlag, New York, 1986.

Department of Mathematics, University of British Columbia, Vancouver, BC V6T 1Z2, CANADA

E-mail address: reichst@math.ubc.ca

$U R L$ : www.math.ubc.ca/ $\sim$ reichst 